\title{
Kidney graft dysfunction in a patient with SARS-CoV-2 pneumonia - Not everything is COVID 19
}

\author{
Cátia Figueiredo $0^{1, *}$, Patrícia Valério ${ }^{2, *}$, Helena Sousa 3,4 , Mário Góis ${ }^{3,4}$, Fernando Nolasco 4 \\ * Equally contributing first authors \\ ${ }^{1}$ Nephrology Department, Hospital de Torres Novas - Centro Hospitalar do Médio Tejo \\ 2 Nephrology Department, Hospital de São Bernardo - Centro Hospitalar de Setúbal; \\ ${ }^{3}$ Nephrology Department, Hospital Curry Cabral - Centro Hospitalar Universitário de Lisboa Central \\ ${ }^{4}$ Laboratory of Renal Morphology, Nephrology Department, Hospital Curry Cabral - Centro Hospitalar Universitário de Lisboa Central
}

We present a case of a 46-year-old African male with chronic kidney disease secondary to hypertensive nephropathy, in hemodialysis for five years. Past medical history included hypertension with hypertensive cardiomyopathy, obesity, and hepatitis B virus infection diagnosed in 2012, treated with tenofovir since then.

In May 2020, the patient underwent a deceased-donor kidney transplantation (KT) with five human leukocyte antigen (HLA) mismatches (two HLA class I and three HLA class II), with no donor-specific antibodies (DSAs), and cold ischemia of 11 hours and 30 minutes. He had no history of previous KT.

The induction immunosuppression used was thymoglobulin for 7 days, followed by tacrolimus, mycophenolate mofetil (MMF) and prednisolone. The dose was reduced at $6^{\text {th }}$ day because of leukopenia total cumulative dose of $1040 \mathrm{mg}$. Tenofovir was changed to entecavir (0.5 mg once a week).

One week after KT, he was submitted to a protocol biopsy, which showed acute tubular necrosis (ATN). The early post-transplant period was characterized by a slow recovery of graft function, with a serum creatinine ( $\mathrm{SCr}$ ) nadir of $1.7 \mathrm{mg} / \mathrm{dL}$.

In the following months, the patient developed medical complications. In the first few weeks, he was diagnosed with new-onset diabetes (NODAT) and insulin therapy was initiated. One month later, he developed an acute allograft disfunction, with a $\mathrm{SCr}$ of $4.0 \mathrm{mg} / \mathrm{dL}$ with preserved urine output. A kidney biopsy was performed, revealing extensive ATN. The etiological study was inconclusive, but the patient improved with fluid therapy.

In the second month post-KT, he presented detectable BK polyoma virus (BK), with a viremia of 320 copies/mL. Immunosuppression was adjusted, with MMF dose reduction. At that time, and after urinary stent removal, a moderate hydronephrosis (without ureter dilatation) and a small peri-graft collection were identified.

One month later, the patient was hospitalized with a SARS-CoV2 pneumonia. Given both infectious conditions (BK viremia and SARSCoV2 pneumonia), the immunosuppression was carefully managed. Initially, prednisolone dose was increased and converted to methylprednisolone (maximum dose of methylprednisolone was $80 \mathrm{mg} /$ day) in the context of respiratory failure secondary to SARS-CoV2 infection. Simultaneously tacrolimus dose was reduced and MMF was discontinued. Over the next few days, BK viremia continued to increase, reaching a peak of 16630 copies/mL. Methylprednisolone was converted to prednisolone $20 \mathrm{mg} /$ day and dose was tapered.

During hospital stay, the graft kidney function deteriorated (maximum SCr of $10.6 \mathrm{mg} / \mathrm{dL}$ ). A new graft ultrasound revealed a worsening hydronephrosis, maintaining the previously identified collection. Given the impossibility of carrying out a graft biopsy, it was decided to start intravenous immunoglobulin (IVIg) $(2 \mathrm{~g} / \mathrm{Kg}$ ), during 3 days. However, the patient developed anuria and required hemodialysis (2 sessions). A nephrostomy was placed and graft function improved progressively, with $\mathrm{SCr}$ stabilization in 3.5-4.0 mg/dL (basal SCr level $2.5 \mathrm{mg} / \mathrm{dL}$ ). The peri-graft collection was subsequently drained.

After these procedures and considering clinical and analytical findings (see Graphic 1), a graft biopsy was performed.

\section{Graph 1}

BK viremia and serum creatinine evolution.

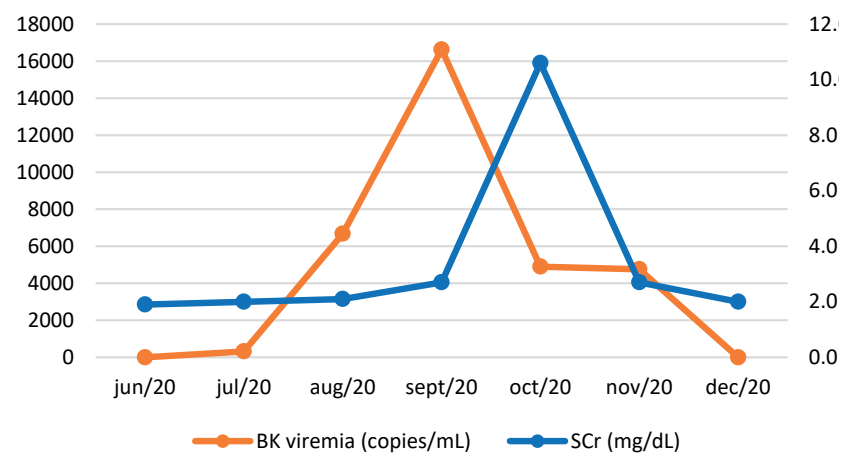

1. What are the most likely diagnoses, considering the clinical history and presentation?

In the first year post-KT, infections and allograft rejections are two of the most common complications. During this period, an aggressive immunosuppression therapy is needed because the likelihood of rejection is highest. After the first year, rejection is typically from noncompliance or overaggressive reduction of immunosuppression. ${ }^{1}$

Considering the case of our patient, an infectious disease and allograft rejection are the most likely diagnoses.

The sustained BK viremia during several weeks and the progressive deterioration of graft function, despite immunosuppression dose 
reduction, is a typical indicator of $\mathrm{BK}$ nephropathy (BKN). Its incidence is highest in the first two to six months post-KT, and it is associated with the highest intensity of immunosuppression, when the patient still is under the effect of induction therapy, which in the case of our patient was thymoglobulin. ${ }^{2}$

Allograft rejection becomes a potential threat when immunosuppression is reduced to deal with infectious complications such as $B K N^{1,2}$ Acute graft rejection is an acute deterioration in graft function associated with specific pathologic changes in the graft. It should be suspected in patients with new increase in $\mathrm{SCr}$, worsening hypertension or proteinuria $>1 \mathrm{~g} /$ day, but the final diagnosis is only obtained with graft biopsy. It allows an accurate diagnosis, distinguishing between T cell-mediated rejection (TCMR) and antibody-mediated rejection (ABMR), and also evaluates the degree of rejection severity and kidney damage. ${ }^{3}$

We could also consider a graft dysfunction associated to COVID-19 infection. It can manifest as AKI, although it remains unclear if $\mathrm{SCr}$ elevation is due to hemodynamic changes and cytokine release, or if the virus also leads to direct cytotoxicity. The histopathological changes are not yet completely understood. ${ }^{4}$

In either case, a graft kidney biopsy is essential to establish the most feasible diagnosis and to predict the prognosis. Although biopsy remains the gold standard for diagnosis of both BKN and graft rejection, the pathological findings can be similar, requiring ancillary tests for distinction.

\section{What is the final diagnosis, considering the histopathological findings?}

The graft kidney biopsy contained 2 fragments: a long medullar fragment, and a smaller one with capsule and cortex, presenting 2 glomeruli and no vessels. In light microscopy, the glomeruli were normal. The cortical fragment presented disseminated interstitial

Figure 1

PAS stain, 200x magnification.

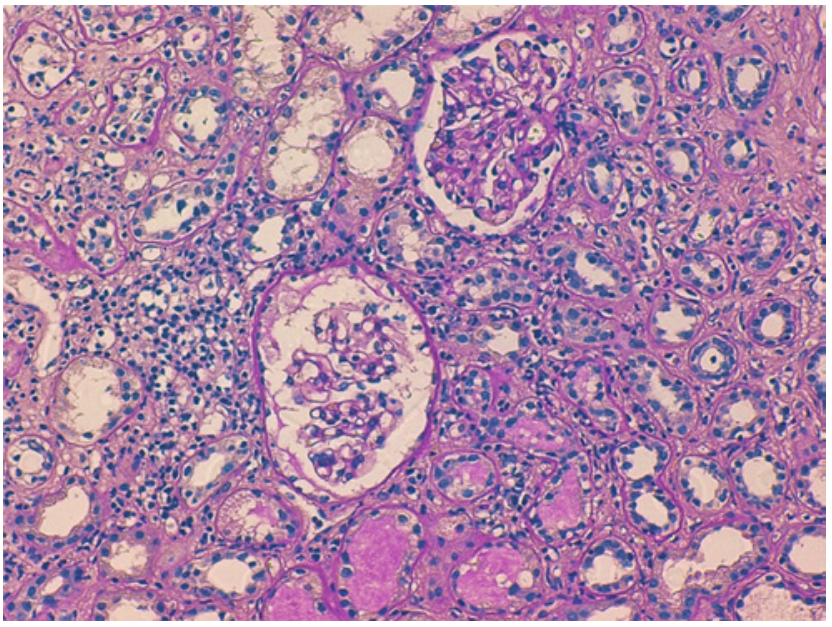

inflammation, with mononuclear inflammatory infiltrate (Figures 1 and 2 ) and extensive interstitial fibrosis (Banff score: $\mathrm{ci}=3$ ) and tubular atrophy (Banff score: $\mathrm{ct}=3$ ). The affected tubular cells presented with anisonucleosis and hyperchromasia (Figure 3).

Immunofluorescence was also negative (IgA, IgM, IgG, C3 and C4d). SV40 staining (immunohistochemistry) was requested, with a positive polyomavirus replication/load level $(p v l=2)$ (Figure 4$)$. The histopathological findings of the graft biopsy made the diagnosis of BKN class 2 (Banff score: pvl2 + ci3).

Since BK preferentially infects epithelial cells from collecting ducts, kidney medullary involvement often predominates. Histopathological changes are compatible with tubulointerstitial nephritis, namely: ${ }^{5}$

\section{Figure 2}

Masson trichrome stain, 100x magnification.

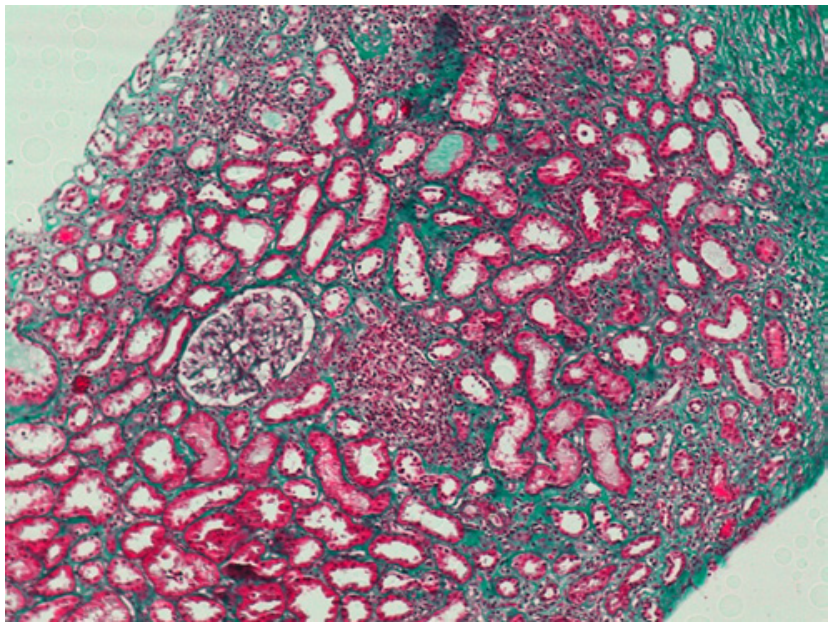

\section{Figure 3}

Hematoxylin and eosin stain, 400x magnification.

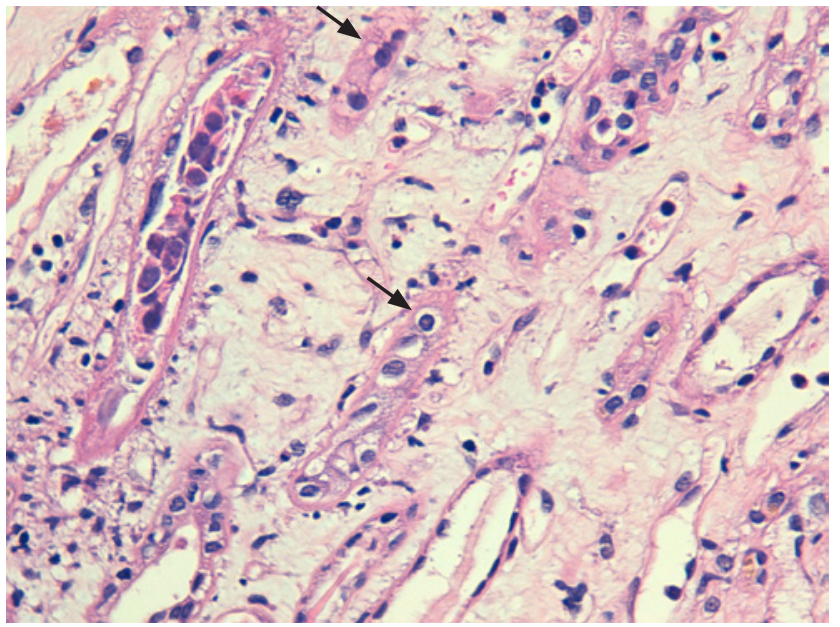




\section{Figure 4}

Immunochemistry for SV40: positive.

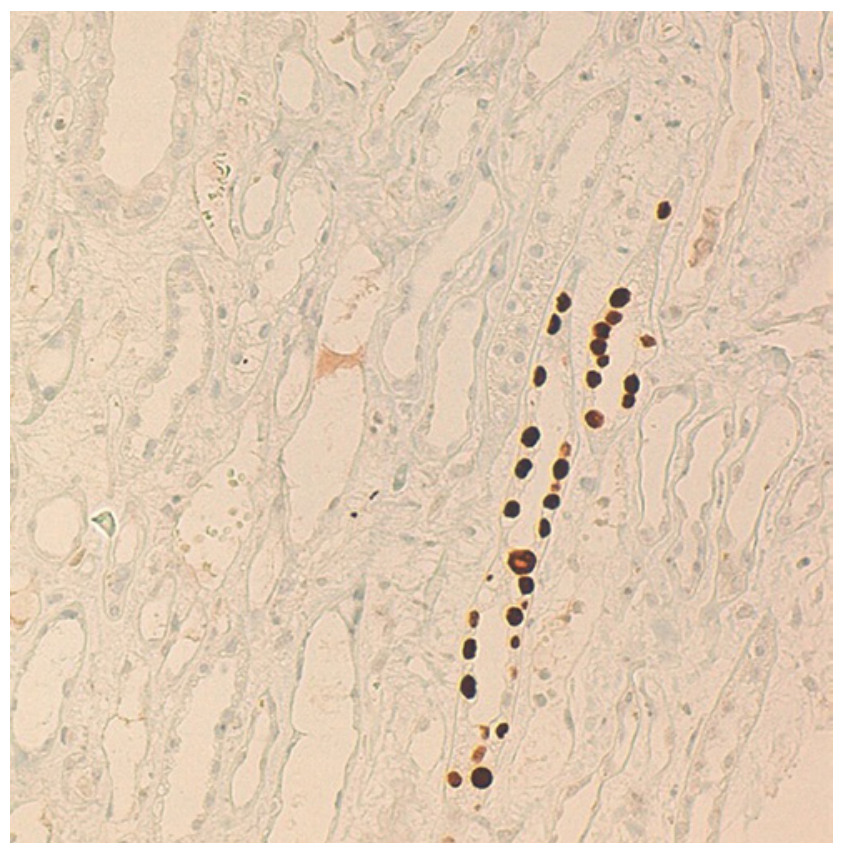

- Tubular injury, with tubular cell apoptosis, cell drop out, desquamation, and flattened epithelial lining;

- Tubulitis, demonstrated by lymphocyte infiltration of the tubular basement membrane;

- Interstitial mononuclear or polymorphonuclear cell infiltrates in the areas of tubular damage.

The infected cells may present anisonucleosis, hyperchromasia, and chromatin clumping. On electronic microscopy, intranuclear basophilic viral inclusions without a surrounding halo may be observed. Areas of tubular atrophy and interstitial fibrosis are usually proportional to the chronicity of the infection. ${ }^{5}$

The most recent classification for PVN are the Banff criteria ${ }^{6}$, which include the degree of tubular viral load (pvl score) and the extent of interstitial fibrosis (ci score). Grade 1 represents early disease with a minor degree of tubular involvement. Grade 2, which was the case of our patient, has a wide spectrum of features, with numerous viral inclusions, tubular necrosis, tubulointerstitial inflammation and $<50 \%$ of fibrosis. Finally, grade 3 is associated with worse outcomes, with sclerotic lesions and $>50 \%$ of fibrosis characterized.

Identification of the virus in the tissue is essential, because light microscopy characteristics, such as interstitial inflammation and tubulitis in the cortex, may also suggest an acute rejection. However, TCMR do not present infected epithelium by BK, a distinctive feature from BKN. Thus, a positive immunohistochemistry test using antibodies directed specifically against BK or against the cross-reacting SV40 large $T$ antigen allows for the definitive diagnosis of BKN and distinguished it from acute rejection. Positive SV40 staining is useful because it presents a specificity of almost $100 \%$ for PVN, although it does not distinguish between BK and JC polyomavirus. ${ }^{6}$

\section{What are the major risks factors for BKN development?}

BK is a ubiquitous infectious agent, causing a latent infection of the kidney epithelium, with reactivation during immunosuppression. Clinically, it may cause nephropathy in KT patients, usually associated with ureteric stenosis and consequently hydronephrosis (as shown in this case), an important cause of allograft function loss. ${ }^{5}$

The degree of immunosuppression, mainly suppression of cellular immunity, is the major risk factor for infection. Consequently, polyomavirus replication rates are higher in the first months after KT. Other risk factors associated with infection are donor seropositivity and allograft rejection. Both viruria and viremia precede the infection, but viremia is more specific for BKN.

\section{How should this patient be managed?}

Disease control is possible through a virus-specific immune response. Therefore, reduction of immunosuppression is essential to manage virus replication.

No specific immunosuppressive drug or regimen has been definitively associated with clinically significant polyomavirus infection. However, several studies have suggested an increased relative risk with calcineurin inhibitors, particularly tacrolimus. Mammalian target of rapamycin (mTOR) inhibitors are probably associated with a lower relative risk. The optimal approach to reduce immunosuppression remains ill-defined. Protocols may vary among transplantation centers, and they are often individualized. A frequently approach is reduction of MMF, followed by calcineurin inhibitors (or the inverted approach). During this process, a close monitoring of these patients is essential to prevent allograft rejection. ${ }^{5}$

Several medical approaches have already been proposed to avoid or treat infection, namely cidofovir, fluoroquinolones or leflunomide, but none of them has demonstrated a clear benefit.

IVIg preparations, which were used in this case, have high titers of neutralizing antibodies to BK, but there is limited data suggesting a possible benefit. ${ }^{2,5}$

Targeted approaches to BKN are still lacking, but a protocoled screening and reduction of immunosuppression has already been shown to be effective in the decrease of BKN incidence.

Our patient was co-infected with BK and SARS-CoV2 and, at that time, COVID-19 protocol at our hospital relied on high doses of corticosteroids. The situation was highly complex to handle because of the two infectious conditions, requiring an opposite management of corticosteroids. The option was for high doses of methylprednisolone and prednisolone, leading to the viremia peak (16630 copies $/ \mathrm{mL}$ ), despite MMF discontinuation on hospital admission. After that, corticosteroids were reduced to the minimum possible. During this period, tacrolimus levels were strictly monitored. 


\section{What is our patient's prognosis?}

Without resolution of infection, progressive renal allograft dysfunction and graft loss can ensue over a period of months. Distinguishing BKN from graft rejection is essential, because the treatment for presumed rejection relies on immunosuppression increase, a strategy that may lead to allograft loss if BKN is present.

Four months after the initial BK viremia detection, the number of virus copies was decreasing, with complete resolution within 10 weeks. At that time, $\mathrm{SCr}$ stabilized at $2.0 \mathrm{mg} / \mathrm{dL}$ and triple immunosuppression (prednisolone, tacrolimus and MMF) was reintroduced.

Disclosure of potential conflicts of interest: none declared

\section{References}

1. Voora S, Adey D. Management of Kidney Transplant Recipients by General Nephrologists: Core Curriculum 2019. Am J Kidney Dis. 2019;73(6):866-879.

2. Fishman J. Infection in solid-organ transplant recipients. N Engl J Med. 2007;357(25):2601-2614.

3. Nankivell B, Alexander S. Rejection of the kidney allograft. N Engl J Med. 2010;363(15):1451-1462.

4. Kant S, Menez S, Hanouneh M, Fine D, Crews D, Brennan D et al. The COVID-19 nephrology compendium: AKI, CKD, ESKD and transplantation.BMC Nephrol. 2020; 27;21(1):449.

5. Kotla S, Kadambi P, Hendricks A, Rojas R. BK polyomavirus-pathogen, paradigm and puzzle. Nephrol Dial Transplant. 2021; 29;36(4):587-593

6. B. Kopp J. Banff classification of polyomavirus nephropathy: A new tool for research and clinical practice. J Am Soc Nephrol. 2018;29(2):354-355.

\section{Correspondence to:}

Mário Góis, MD

Laboratory of Renal Morphology Hospital Curry Cabral

Centro Hospitalar de Lisboa Central, Lisboa, Portugal

E-mail: mario.gois@chlc.min-saude.pt 